\title{
Defining the Mer1 and Nam8 meiotic splicing regulons by cDNA rescue
}

\author{
ZHICHENG R. QIU, ${ }^{1}$ BEATE SCHWER, ${ }^{2}$ and STEWART SHUMAN ${ }^{1,3}$ \\ ${ }^{1}$ Molecular Biology Program, Sloan-Kettering Institute, New York, New York 10065, USA \\ ${ }^{2}$ Department of Microbiology and Immunology, Weill Cornell Medical College, New York, New York 10065, USA
}

\begin{abstract}
Meiosis-specific pre-mRNA splicing in budding yeast embraces multiple pre-mRNA targets grouped into regulons defined by their genetic requirements for vegetatively optional splicing factors (e.g., splicing enhancer Mer1 and the U1 snRNP subunit Nam8) or snRNA modifications (trimethylguanosine caps). Here, we genetically demarcate a complete meiotic splicing regulon by the criterion of cDNA bypass of the requirement for the governing splicing regulators to execute sporulation. We thereby show that the Mer1 and Nam8 regulons embrace four essential pre-mRNAs: MER2, MER3, SPO22, and AMA1. Whereas Nam8 also regulates $\mathbf{P C H} 2$ splicing, $\mathrm{PCH} 2 \mathrm{cDNA}$ is not needed for sporulation by nam8D diploids. Our results show that there are no essential intron-containing RNAs missing from the known roster of Mer1 and Nam8 targets. Nam8 is composed of three RRM domains, flanked by N-terminal leader and C-terminal tail segments. We find that the RRM2 and RRM3 domains, and their putative RNA-binding sites, are essential for yeast sporulation, whereas the leader, tail, and RRM1 modules are not.
\end{abstract}

Keywords: AMA1; MER2; MER3; SPO22

\section{INTRODUCTION}

The budding yeast Saccharomyces cerevisiae, which has been a powerful model system to elucidate the composition and function of the core pre-mRNA splicing machinery (Fabrizio et al. 2009), relies heavily during meiosis on controlling the mode of alternative splicing known as intron retention (Nilsen and Graveley 2010). The yeast meiotic developmental program entails a shift in the processing patterns of target pre-mRNAs from a vegetative "off" state, in which single introns are included, to a meiotic "on" state, in which the target introns are removed. The initial discovery of Mer1regulated meiotic splicing of the MER2 pre-mRNA and the appreciation that Mer1 functions together with Nam8 to compensate for inherently weak introns are landmarks in the field (Engebrecht et al. 1991; Nandabalan et al. 1993; Spingola and Ares 2000).

The 523-aa Nam8 protein is a subunit of the yeast U1 snRNP (Gottschalk et al. 1998). Nam8 contains three tandem RRM domains, and is inessential for yeast mitotic growth, but is essential for yeast sporulation because it promotes splicing of a set of $\mathrm{mRNAs}$ that encode proteins required for

\footnotetext{
${ }^{3}$ Corresponding author.

E-mail s-shuman@ski.mskcc.org.

Article published online ahead of print. Article and publication date are at http://www.rnajournal.org/cgi/doi/10.1261/rna.2792011.
}

meiotic recombination and cell division (Nakagawa and Ogawa 1997; Spingola and Ares 2000). Nam8-dependent splicing of four meiotic mRNAs-AMA1, MER2, MER3, and SPO22-is activated by the meiotic splicing regulator Mer1 (Engebrecht et al. 1991; Nakagawa and Ogawa 1999; Cooper et al. 2000; Spingola and Ares 2000; Munding et al. 2010; Qiu et al. 2011a). Mer1 is a 270-aa polypeptide composed of two functional modules: a C-terminal $\mathrm{KH}$ domain implicated in RNA binding and an N-terminal domain that interacts with the spliceosome and promotes splicing of target mRNAs (Spingola et al. 2004; Qiu et al. 2011a). Mer1 is produced only in meiotic cells under the control of the meiotic transcription factor Ime1 (Engebrecht and Roeder 1990). Mer1 activates splicing by binding to an intronic splicing enhancer sequence ( $5^{\prime}$-AYACCCUY-3') present in the AMA1, MER2, MER3, and SPO22 pre-mRNAs (Spingola and Ares 2000; Qiu et al. 2011a). Mer1 bound to the intronic enhancer is thought to promote assembly of the U1 and U2 snRNPs on the pre-mRNA (Balzer and Henry 2008). Transcripts subject to Mer1/Nam8 splicing regulation have suboptimal 5' splice sites that dictate their reliance on the otherwise inessential Mer1 and Nam8 splicing factors (Nandabalan et al. 1993; Spingola et al. 2004; Qiu et al. 2011a). Based on the shared genetic requirements in cis and trans for their splicing, it is posited that AMA1, MER2, MER3, and SPO22 comprise a meiotic splicing "regulon." 
Recent studies have identified the $\mathrm{PCH} 2$ and $S A E 3$ premRNAs as targets of regulated meiotic splicing governed by different splicing factors and RNA features (Qiu et al. 2011a,b). The PCH2 and SAE3 pre-mRNAs have consensus 5' splice sites, lack intronic Mer1 enhancer elements, and do not rely on Mer1 for their splicing in vivo (Qiu et al. 2011a,b). SAE3 splicing during meiosis depends on Tgs1, the methyltransferase enzyme that forms the TMG cap found on the spliceosomal U1, U2, U4, and U5 snRNAs (Qiu et al. 2011b). Tgs1 methyltransferase activity is inessential for vegetative growth of $S$. cerevisiae, but is required for sporulation (Qiu et al. 2011b). The dependence of SAE3 splicing on TMG caps is dictated by its nonconsensus intron branchpoint sequence and a masking secondary structure at the branchpoint (Qiu et al. 2011b). SAE3 splicing does not rely on Nam8. In contrast, splicing of $\mathrm{PCH} 2$ pre-mRNA during meiosis is codependent on Nam8 and Tgs1. For PCH2, a long 5' exon and a nonconsensus intron branchpoint dictate Nam8 and Tgs1 dependence (Qiu et al. 2011a,b). Thus, Nam8 participates in two distinct meiotic splicing regulons.

These findings highlight new modes of tunable splicing during development and the presence of at least three genetically distinct meiotic splicing regulons in budding yeast. Two key questions arise. Are there additional yeast meiotic splicing regulons? Do we have a complete roster of the relevant pre-mRNA targets for any of the several meiotic regulons we know about? In the present study, we address the second question.

Our working definition of a "complete" meiotic splicing regulon is the ability to rescue the sporulation defect of a deletion mutant of the splicing controller by expressing intronless (cDNA) versions of the set of essential meiotic RNAs that are targeted by that controller. Prior efforts to accomplish this for Mer1 and Nam8 have not succeeded, insofar as expression of intronless $c M E R 2$, intronless $c M E R 3$, or a combination of $c M E R 2$ plus $c M E R 3$, did not fully rescue the sporulation defects of nam $8 \Delta$ or mer $1 \Delta$ diploids (Engebrecht et al. 1991; Nakagawa and Ogawa 1999). The results of our survey of all presently identified meiotic spliced mRNAs (Juneau et al. 2007) for splicing deficiencies in nam $8 \Delta$ diploids during attempted sporulation, and of specific tests of Mer1-dependence (Qiu et al. 2011a), suggest that we might indeed be able to delineate complete Mer1 and Nam8 and regulons by complementation with a wider net of cDNAs. Our approaches, and the issues at stake, are described below.

\section{RESULTS AND DISCUSSION}

\section{Defining the Mer1 regulon}

Our results suggested a four-membered Mer1 regulon embracing MER2, MER3, AMA1, and SPO22 (Qiu et al. 2011a). To test its completeness, we introduced pairs of intronless cDNAs into inessential chromosomal loci of mer1s SK1 haploids of opposite mating types (cMER2 plus $c M E R 3$ in

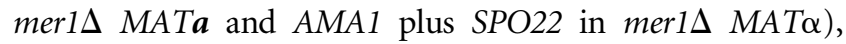
mated them to generate mer $1 \Delta$ diploids expressing all four intronless Mer1 targets, and then assessed their sporulation efficiency. In parallel, we analyzed wild-type SK1 (positive control), mer1s SK1 with no cDNAs (negative control), wild-type SK1 expressing pairs or all four of the cDNAs, and mer1 $\Delta$ SK1 expressing only two of the cDNAs. If we could bypass the mer $1 \Delta$ mutation by any of these maneuvers, then the results would define the regulon as complete (at least with respect to targets essential for sporulation). If we could not obtain bypass, then we would surmise that: (1) either there are additional RNA targets of Mer1 splicing control not yet identified, or (2) Mer1 performs extra functions in the sporulation program unrelated to meiotic pre-mRNA splicing.

Figure 1 shows the kinetics of sporulation of wild-type SK1 diploids with or without meiotic cDNA expression. Wild-type SK1 with no cDNAs synchronously formed asci between 8 and $12 \mathrm{~h}$ after transfer to sporulation medium and attained $95 \%$ sporulation efficiency at $24 \mathrm{~h}$. Coexpression of the MER2, MER3, AMA1, and SPO22 cDNAs under the control of their native promoters caused a modest but reproducible reduction in the rate and extent of sporulation $(70 \%$ at $24 \mathrm{~h})$, but did not affect the lag time between induction of meiosis and the initial appearance of four-spore asci (Fig. 1, top) or the viability of the spores contained within asci, as gauged by tetrad dissection (Table 1). Spore viability was $98 \%$ in the wild-type strain versus $97 \%$ in the
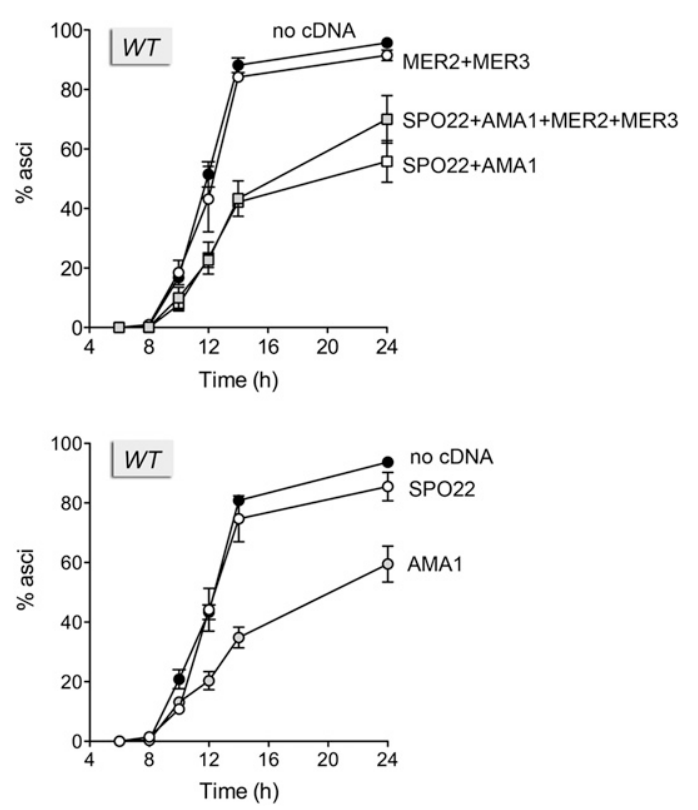

FIGURE 1. Effects of ectopic cDNA expression on sporulation by wild-type diploids. Wild-type diploids with the indicated cDNAs integrated at the chromosomal LEU2 or URA3 locus were examined by light microscopy at the indicated times after transfer to sporulation medium. The percentages of the cell population comprising fourspore asci are plotted as a function of time. Each datum is the average of three independent experiments \pm SEM. 
TABLE 1. Meiotic regulon cDNA expression does not affect spore viability

\begin{tabular}{ll}
\hline Strain & Spore viability \\
\hline$W T$ & $98 \%(164 / 168)$ \\
$W T+$ cMER2 CMER3 CAMA1 CSPO22 & $97 \%(166 / 172)$ \\
mer1 + cMER2 CMER3 CAMA1 CSPO22 & $94 \%(166 / 176)$ \\
nam8D + cMER2 CMER3 CAMA1 CSPO22 & $97 \%(179 / 184)$ \\
\hline
\end{tabular}

Cultures of the indicated diploids strains were evaluated $24 \mathrm{~h}$ after induction of meiosis. Spore viability was gauged by tetrad dissection and quantified as the percent of spores germinating to form macroscopic colonies after incubation for $2 \mathrm{~d}$ at $30^{\circ} \mathrm{C}$ on YPD agar medium. The values in parenthesis are the numbers of viable spores over the total number of spores harvested.

wild-type strain coexpressing the four cDNAs. Whereas pairwise coexpression of MER2 and MER3 cDNAs had little or no impact on sporulation kinetics or efficiency at $24 \mathrm{~h}$ (92\%), the pairwise coexpression of AMA1 and SPO22 cDNAs elicited decrements in the rate and extent of sporulation ( $56 \%$ at $24 \mathrm{~h}$ ) that echoed the effects of expressing all four cDNAs (Fig. 1, top). To investigate this phenomenon further, we constructed wild-type SK1 diploids expressing single AMA1 or SPO22 cDNAs and monitored their sporulation kinetics in parallel with a "no cDNA" wild-type control. Whereas the SPO22 cDNA had little impact on sporulation rate or yield $(86 \%$ at $24 \mathrm{~h}$ vs. $93 \%$ for no cDNA), the AMA1 cDNA slowed sporulation and reduced 24-h efficiency to $59 \%$ (Fig. 1, bottom). We surmise that precocious expression of $A M A 1$ is moderately deleterious to the yeast meiotic program (discussed further below).

mer1s diploids were grossly defective in sporulation, as expected, yielding only $6 \%$ asci after $24 \mathrm{~h}$ (Fig. 2, top). The key finding was that coexpression of the MER2, MER3, $A M A 1$, and SPO22 cDNAs in mer1 $\triangle$ diploids reversed the defect and restored sporulation efficiency to $73 \%$ (Fig. 2, top), comparable to the $70 \%$ level attained by wild-type diploids coexpressing the four cDNAs. Spore viability was $94 \%$ in the mer $1 \Delta$ strain coexpressing the four cDNAs (Table 1). Pairwise expression of MER2 and MER3 cDNAs in mer1 $1 \Delta$ diploids conferred no benefit ( $6 \%$ sporulation at $24 \mathrm{~h}$ ). However, a partial gain-of-function to $27 \%$ sporulation was attained by coexpressing AMA1 and SPO22 cDNAs in mer1 1 diploids (Fig. 2, top). These results establish that the Mer1 splicing regulon embraces the MER2, MER3, AMA1, and SPO22 premRNAs and no other meiotically essential intron-containing transcripts. They also suggest a hierarchy of importance of regulon members during the meiotic program, whereby feeble splicing of AMA1 and/or SPO22 has a greater impact than inefficient splicing of MER2 and MER3.

\section{Defining the Nam8 regulon}

The U1 snRNP subunit Nam8 controls splicing of the Mer1 regulon plus the $\mathrm{PCH} 2$ pre-mRNA (Qiu et al. 2011a). Because a pch $2 \Delta$ mutant is competent for sporulation in an otherwise wild-type background (San-Segundo and Roeder 1999), we tested whether the expression of cDNA for the other four Nam8 targets, pairwise or en bloc, could rescue the severe sporulation defect of a nam $8 \Delta$ SK1 diploid ( $1 \%$ asci at $24 \mathrm{~h}$ ). The results are shown in Figure 2 (bottom). Again, the instructive finding was that coexpression of the MER2, MER3, AMA1, and SPO22 cDNAs in nam8D diploids restored sporulation efficiency to $66 \%$. Spore viability was $97 \%$ in the nam $8 \Delta$ strain coexpressing the four cDNAs (Table 1). Whereas pairwise expression of MER2 and MER3 cDNAs in nam $8 \Delta$ diploids was inconsequential ( $2 \%$ sporulation), coexpression of AMA1 and SPO22 cDNAs elicited a modest recovery (15\% sporulation). We conclude that the Mer1 and Nam8 splicing regulons embrace the same set of four essential meiotic pre-mRNAs: MER2, MER3, $A M A 1$, and SPO22.

\section{Domain requirements for Nam8 function in meiosis}

Nam8 is a 523-aa polypeptide that includes three RRM domains (Fig. 3). The closely spaced RRM1-RRM2 tandem unit is preceded by a 40 -aa $\mathrm{N}$-terminal leader peptide and separated from the downstream RRM3 domain by a hydrophilic 63-aa inter-RRM linker peptide rich in Ser, Asn, and Gln. Distal to RRM3 is a hydrophilic 130-aa C-terminal extension that is also rich in Ser, Asn, and Gln (Fig. 3A). An alignment of the primary structures of $S$. cerevisiae and Ashbya gossypii Nam8 helped guide the design of serial
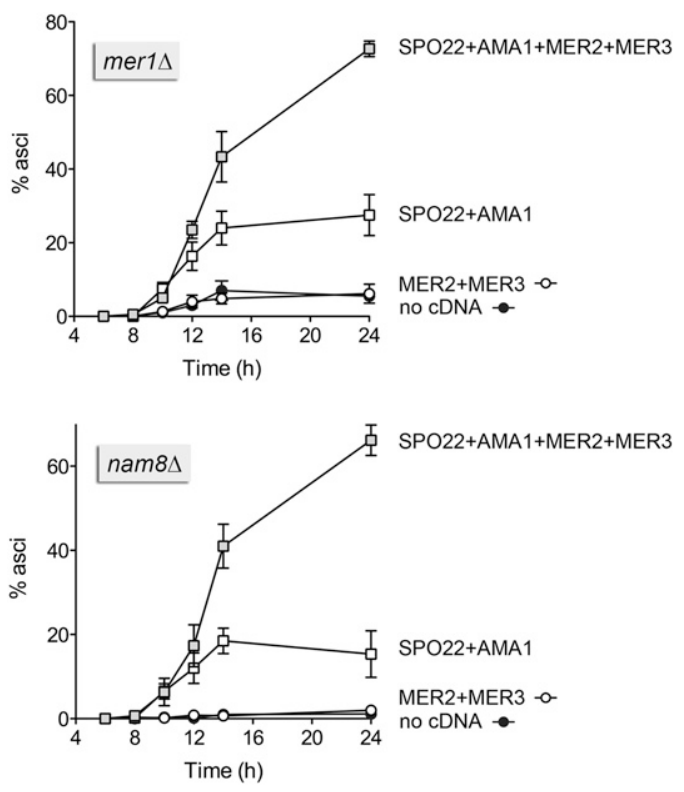

FIGURE 2. cDNA rescue defines the Mer1 and Nam8 splicing regulons. mer $1 \Delta$ and nam $8 \Delta$ diploids with the indicated cDNAs integrated at the chromosomal LEU2 or URA3 locus were examined by light microscopy at the indicated times after transfer to sporulation medium. The percentages of the cell population comprising fourspore asci are plotted as a function of time. Each datum is the average of three independent experiments \pm SEM. 

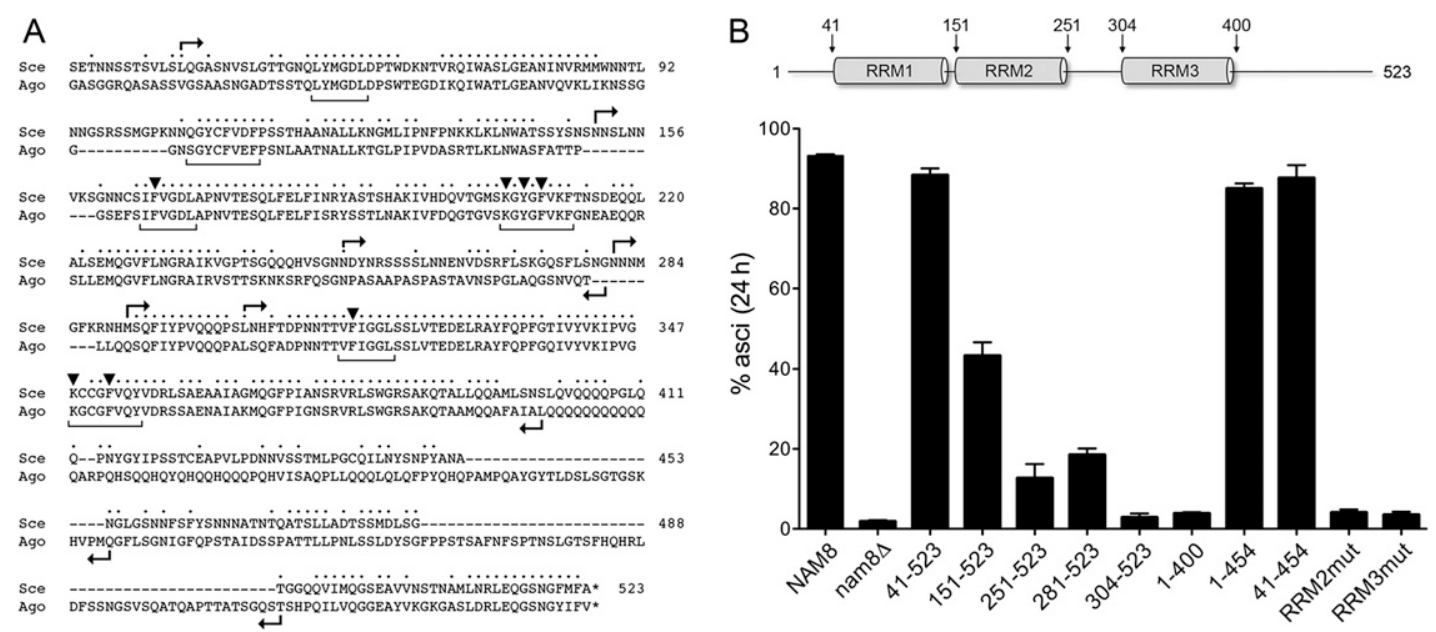

FIGURE 3. Nam8 domain organization and requirements for sporulation. (A) The amino acid sequence of S. cerevisiae (Sce) Nam8 from residues 28-523 is aligned to the sequence of A. gossypii (Ago) Nam8. Positions of amino acid side chain identity/similarity are denoted by $\bullet$ above the sequence. RNP2 and RNP1 motifs of the RRMs are indicated by brackets under the sequence. Positions within the motifs of the RRM2 and RRM3 domains that comprise the putative RNA-binding surface and were subjected to alanine cluster mutagenesis are denoted by $\boldsymbol{\nabla}$ above the sequence. Forward and reverse arrows indicate the boundaries of the $\mathrm{N}$ - and C-terminal truncations of Nam8, respectively. ( $B$, top) Schematic representation of Nam8 domain organization; the RRMs are depicted as cylinders. (Bottom) nam $8 \Delta$ diploids with the indicated NAM8 alleles integrated at the chromosomal LEU2 locus were examined by light microscopy at $6,8,10,12,14$, and $24 \mathrm{~h}$ after transfer to sporulation medium. The percentages of the cell population comprising four-spore asci were gauged. The bar graph shows the extents of sporulation at $24 \mathrm{~h}$. Each datum is the average of three independent experiments \pm SEM.

$\mathrm{N}$ - and C-terminal truncations of S. cerevisiae Nam8 at the putative domain (or subdomain) boundaries indicated by the forward and reverse arrowheads in Figure 3A. In a previous study, we tested the truncated NAM8 alleles for their ability to support vegetative growth of nam $8 \Delta$ cells in a variety of synthetic lethal genetic backgrounds, e.g., with $\operatorname{tgs} 1 \Delta$, lea $1 \Delta$, mud1s, and mud2s (Qiu et al. 2011a). We found that the leader, tail, and RRM1 were dispensable for Nam8 vegetative functions. In contrast, deleting RRM1 and RRM2 together (or together with the inter-RRM linker) exerted different effects in different synthetic backgrounds, ranging from retention of full or partial activity to ablation of function, as gauged by cell growth.

Our aim in the present study was to directly probe the structure-function relationships of Nam8 in meiosis by introducing truncated and mutated NAM8 alleles into SK1 nam $8 \Delta$ diploids and examining their kinetics and efficiencies of sporulation. The results are summarized in bar graph format Figure 3B, which shows the percents of four-spore asci $24 \mathrm{~h}$ after transfer of the diploids to sporulation medium. The sporulation efficiencies of the NAM8 and nam $8 \Delta$ strains were $93 \%$ and $2 \%$, respectively. Whereas deletion of the $\mathrm{N}$-terminal leader in NAM8-(41-523) had little or no effect on sporulation efficiency (88\%), further deletion of the RRM1 module in NAM8-(151-523) reduced sporulation efficiency to $43 \%$ (Fig. 3B). Extending the N-terminal deletions to include RRM2 and part of the inter-RRM linker in NAM8-(251-523) and NAM8-(281-523) elicited more severe decrements, to $13 \%$ and $18 \%$ sporulation, respectively. The incremental deletion of all of the inter-RRM linker up to the proximal margin of RRM3 in NAM8-(304-523) supported only 3\% sporulation, thus mimicking the nam $8 \Delta$ null strain. We conclude that RRM2 and the inter-RRM segment are critical for Nam8 meiotic function and that the domain requirements for Nam8 activity during yeast meiosis are more stringent than during vegetative growing of several Nam8-dependent strains.

Whereas truncation of the Nam8 C terminus to position 454 had little or no impact [ $85 \%$ sporulation of the NAM8(1-454) strain], further deletion to position 400 virtually abolished Nam8 meiotic function $[4 \%$ sporulation by NAM8-(1-400) diploids] (Fig. 3B). Combining the "active" $\mathrm{N}$-terminal leader and C-terminal trailer peptide deletions in mutant allele NAM8-(41-454) resulted in retention of full meiotic activity ( $88 \%$ sporulation), signifying that the leader and trailer are not functionally redundant. The effects of C-terminal deletions on Nam8 meiotic functions are concordant with their impact on essential Nam8 activities in vegetative cells (Qiu et al. 2011a). The segment between 401 and 454, deletion of which abolishes all Nam8 functions, meiotic and vegetative, contains a glutamine patch (QVQQQQPGLQQ ${ }^{412}$ ), but is otherwise unremarkable.

\section{Effects of alanine mutations in RRM2 and RRM3 on Nam8 function in meiosis}

The RRM fold comprises a four-stranded $\beta$-sheet flanked on one side by two $\alpha$-helices. RRM domains typically bind RNA along the exposed surface of the $\beta$-sheet opposite the $\alpha$-helices (Sickmier et al. 2006). The central $\beta 1$ and $\beta 3$ 
strands of the sheet (referred to as the RNP2 and RNP1 motifs, respectively, and denoted by brackets under the Nam8 primary structure in Figure 3A) make most of the atomic contacts with bound RNA. Here, we tested the effects of alanine mutations in the putative RNA-binding surfaces of RRM2 and RRM3 in Nam8. The alanine changes were introduced into Nam8-(41-454), a biologically active allele that includes all three tandem RRMs. The Ala-mutant alleles were assayed for complementation of the meiotic defect of the nam8s SK1 diploid strain. With respect to RRM2, we tested a quadruple-alanine substitution (named "RRM2mut" in Fig. 3B) for Phe166 in the $\beta 1$ strand RNP2 motif and for Lys205, Tyr207, and Phe209 in the $\beta 3$ strand RNP1 motif; these side chains ([V] in Fig. 3A) project outward on the surface of the $\beta$-sheet and correspond to residues in canonical RRMs that interact with RNA. In RRM3, we tested a triple-alanine substitution (named "RRM3mut" in Fig. 3B) for putative RNA-binding residues Phe316 in the $\beta 1$ strand and Lys348 and Phe352 in the $\beta 3$ strand ( $[\boldsymbol{\nabla}]$ positions in Fig. 3A). The NAM8-RRM2mut and NAM8-RRM3mut strains failed to sporulate ( $4 \%$ asci at $24 \mathrm{~h}$, mimicking nam $8 \Delta)$. We conclude that the putative RNA-binding surfaces of RRM2 and RRM3 are both essential for Nam8 meiotic function. These results are consistent with the impact of the RRM2mut and RRM3mut alanine substitutions on the efficiency of MER2 pre-mRNA splicing in vegetative yeast cells that ectopically express Mer1 (Qiu et al. 2011a).

\section{The Tgs1 meiotic splicing regulon: More than SAE3}

Yeast $\operatorname{tgs} 1 \Delta$ cells are sporulation incompetent and defective in splicing the $P C H 2$ and SAE3 meiotic pre-mRNAs (Qiu et al. $2011 b)$. The magnitude of the $\mathrm{PCH} 2$ splicing defect during attempted sporulation is similar in $\operatorname{tgs} 1 \Delta$ and nam $8 \Delta$ mutants. As demonstrated above by cDNA rescue of nam $8 \Delta$ sporulation, $\mathrm{PCH} 2$ is not an essential constituent of the Nam8 splicing regulon. Assuming the same is true of the Tgs1 regulon, we attempted to bypass the meiotic requirement for Tgs1 by introducing an intronless SAE3 cDNA into a chromosomal locus of TGS1 and $\operatorname{tgs} 1 \Delta$ SK1 diploids. The kinetics of synchronous sporulation and the yields of four-spore asci at $24 \mathrm{~h}(93 \%-96 \%)$ were similar for TGS1 diploids with and without the SAE3 cDNA (Supplemental Fig. S1). The $\operatorname{tgs} 1 \Delta$ diploids were grossly defective (4\% sporulation efficiency). Expression of the SAE3 cDNA in tgs $1 \Delta$ diploids under the control of the $S A E 3$ promoter resulted in no enhancement of ascus formation at up to $14 \mathrm{~h}$ post-transfer to sporulation medium, but did elicit $16 \%$ sporulation at $24 \mathrm{~h}$ (Supplemental Fig. S1). From this result we surmise that there are additional essential pre-mRNAs comprising the Tgs1 meiotic regulon.

\section{Conclusions and implications}

Regulated pre-mRNA splicing during budding yeast meiosis embraces multiple pre-mRNA targets grouped into different, but partially overlapping regulons defined by their genetic requirements for particular splicing factors or RNA modifications. It is noteworthy that the several proteins that are known to govern meiotic pre-mRNA splicing are optional for splicing during vegetative growth. This scenario likely reflects the prevalence of nonconsensus intronic splicing signals and atypically long $5^{\prime}$ exons among the cadre of intron-containing meiotic genes. Here, we provide a genetic definition of a complete meiotic splicing regulon by the criterion of cDNA bypass of the requirement for the governing splicing regulator(s) to attain efficient execution of the sporulation program. In this way, we show that there are four essential pre-mRNA targets comprising the Mer1 and Nam8 regulons: MER2, MER3, SPO22, and $A M A 1$. Whereas Nam8 also regulates $P C H 2$ splicing in a Mer1-independent manner, $\mathrm{PCH} 2$ is not required for sporulation by otherwise wild-type diploids (San-Segundo and Roeder 1999). In effect, our results show that there are no essential intron-containing RNAs missing from the roster of Mer1 and Nam8 targets.

In contrast, our inability to rescue $\operatorname{tgs} 1 \Delta$ sporulation by provision of a cDNA for $S A E 3$, the essential meiotic transcript for which splicing is most acutely reliant on Tgs1 (Qiu et al. 2011b), argues that the Tgs1 regulon must include other essential meiotic transcripts. In our prior study describing the requirements for Nam8 and Tgs1 for sporulation and meiotic splicing, the criterion applied to designate a pre-mRNA as dependent on Nam8 or Tgs1 was a greater than or equal to fourfold decrement in splicing efficiency in a nam $8 \Delta$ or $\operatorname{tgs} 1 \Delta$ diploid undergoing attempted sporulation vs. the splicing efficiency of the same transcript in NAM8 and TGS1 controls analyzed in parallel (Qiu et al. 2011a,b). In tgs $1 \Delta$ cells, only SAE3 and PCH2 met this fourfold threshold. However, there were three additional essential meiotic transcripts that suffered what we deemed a modest decrement (at least twofold) in their splicing efficiency in $\operatorname{tgs} 1 \Delta$ cells: AMA1, MER3, and MEI4 (Qiu et al. 2011b). Thus, it is conceivable, indeed likely, that inefficient splicing of one or all of this trio of premRNAs (or other Tgs1-dependent transcripts as yet undefined) contributes to the $\operatorname{tgs} 1 \Delta$ sporulation-defective phenotype. Additional rounds of cDNA rescue trials will be required to clarify this issue.

Speculations regarding the prominence of up-regulated splicing during yeast meiosis-by transition from a vegetative "splicing-off" state to a new "splicing-on" modehave focused on its potential to protect vegetative cells from the untimely production of potentially deleterious meiotic proteins (Juneau et al. 2007; Maleki et al. 2007). Maintenance of the splicing-off state in vegetative cells is, in effect, a back-up to the "transcription-off" state of most meiotic intron-containing genes during vegetative growth (Primig et al. 2000; Rabitsch et al. 2001; Munding et al. 2010; Qiu et al. 2011a), MER2 being an exception that is transcribed constitutively (but spliced inefficiently) in 
vegetative cells (Engebrecht et al. 1991). Munding et al. (2010) have articulated a more nuanced view of meiotic splicing regulation as a means to tune the kinetics of the expression of a subset of meiotic genes that are transcriptionally induced during the early phase of the meiotic program. The splicing regulator Mer1 and three of its targets-MER3, SPO22, and AMA1-are coordinately induced early during sporulation via the master meiotic transcription factor Ime1, also entailing relief from vegetative transcriptional repression by Ume6. Because the generation of functional MER3, SPO22, and AMA1 transcripts relies on Mer1-activated splicing of their pre-mRNAs, the production of Mer3, Spo22, and Amal proteins will be delayed (while waiting for Mer1 protein to accumulate) compared with the proteins encoded by Imel-induced genes that either lack introns or have introns that are spliced constitutively (Munding et al. 2010). Whereas deletion of Mer1 and some of its targets results in defective execution of downstream events in the meiotic transcriptional cascade, it was not clear whether removal of the delay in the onset of expression of any of the mRNAs in the Mer1/ Nam 8 regulon would have any consequences. Arguably, this is the key issue concerning the kinetic delay model.

Our studies here establish that expression of an AMA1 cDNA under the control of the AMA1 promoter, a maneuver that will result in precocious appearance of mature AMA1 mRNA early in meiosis (i.e., with no wait for Mer1dependent splicing), exerted a deleterious effect on sporulation efficiency by wild-type yeast. AMA1 was unique among the Mer1/Nam8 regulon components in this respect. The AMA1 effect makes sense, insofar as Amal is a meiosisspecific coactivator of the yeast anaphase-promoting complex (APC, a ubiquitin ligase) that directs the degradation of proteins that inhibit anaphase (Cooper et al. 2000; Oelschlaegel et al. 2005). Meiotic inhibition by $\mathrm{Mnd} 2$ of Ama1-APC activity guards against premature chromosome separation in prophase I (Oelschlaegel et al. 2005; Penkner et al. 2005). We envision that precocious Amal expression early in meiosis results in premature activation of Ama1-APC activity, triggering chromosome separation (and/or other defects) in a fraction of the diploids undergoing meiosis, which leads to the lower yield of four-spore asci that we observe when wild-type yeast express the AMA1 cDNA. Thus, Mer1regulated splicing confers a tangible advantage, likely by delaying the production and action of Amal until the synapsed homologous chromosomes are ready for anaphase.

\section{MATERIALS AND METHODS}

\section{Plasmid constructions}

A DNA fragment containing the MER2 ORF and intron (1.0 kbp) plus $470 \mathrm{bp}$ of upstream $\left(5^{\prime}\right)$ and $328 \mathrm{bp}$ of downstream $\left(3^{\prime}\right)$ chromosomal DNA was amplified by PCR from $S$. cerevisiae genomic DNA using primers that introduced a SacI site at the $5^{\prime}$ end and a NotI site at the $3^{\prime}$ end. The following DNA fragments were amplified similarly by PCR: the MER3 ORF and intron (3.7 $\mathrm{kbp})$ plus $435 \mathrm{bp}$ and 288 bp of $5^{\prime}$ and $3^{\prime}$ flanking genomic DNA with 5' BamHI and 3' SalI sites; the AMA1 ORF and intron (1.9 $\mathrm{kbp}$ ) plus $474 \mathrm{bp}$ and $307 \mathrm{bp}$ of $5^{\prime}$ and $3^{\prime}$ flanking genomic DNA with 5' HindIII site and 3' XhoI sites; the SPO22 ORF and intron (3.0 kbp) plus $441 \mathrm{bp}$ and $307 \mathrm{bp}$ of flanking genomic DNA with $5^{\prime}$ NotI and 3' BamHI sites; and the SAE3 ORF and intron (362 bp) plus $370 \mathrm{bp}$ and $255 \mathrm{bp}$ of flanking genomic DNA with 5' SacI and ' ' HindIII sites. The MER2, MER3, AMA1, SPO22, and SAE3 introns were removed cleanly from their genomic fragments via two-stage overlap extension PCR to generate the respective cDNAs (cMER2, cMER3, cAMA1, cSPO22, and cSAE3) with their genomic DNA flanks and terminal restriction sites. The MER2 and $M E R 3$ cDNAs were restricted at the terminal sites and then inserted in tandem (with $c M E R 2$ upstream of $c M E R 3$ ) into yeast integrative vector pRS305 (LEU2) to yield pRS305-cMER2-cMER3. The AMA1 and SPO22 cDNAs were cleaved at the terminal restriction sites and inserted individually into yeast integrative vector pRS306 (URA3) to yield pRS306-cAMA1 and pRS306-cSPO22. The SPO22 cDNA was also inserted into pRS306-cAMA1 (upstream of $c A M A 1$ ) to yield pRS306-cAMA1-cSPO22. The SAE3 cDNA was terminally restricted and inserted into pRS305 to yield pRS305-cSAE3. To construct pRS305 plasmids bearing wild-type and mutant NAM8 alleles, HindIII-SacI fragments were excised from yeast Nam8 expression vectors described previously (Qiu et al. 2011a) and inserted into pRS305. The inserts of all plasmid clones were sequenced to exclude the acquisition of unwanted mutations during amplification and cloning.

\section{Yeast strains}

The meiosis/sporulation experiments were carried out with isogenic diploids in the SK1 background. The construction of nam $8 \Delta$ and $\operatorname{tgs} 1 \Delta$ haploid strains was described previously (Qiu et al. $2011 \mathrm{a}, \mathrm{b})$. mer1 $\Delta$ strains were generated by replacing the entire MER1 open reading frame with a kanMX cassette in SKY163 (MATa ho::LYS2 lys2 ura3 leu2::hisG) and a natMX cassette in SKY164 (MAT $\alpha$ ho::LYS2 lys2 ura3 leu2::hisG). In brief, the disruption cassettes were constructed by inserting PCR-generated MER1 5'-flanking (620 bp) and 3'-flanking (565 bp) genomic fragments into pUG6 (Wach et al. 1994) and pAG25 (Goldstein and McCusker 1999), so that they flank the kanMX and natMX cassettes, respectively. The integration cassettes were excised and transformed into the haploid SKY163 and SK164 strains. The targeted insertions were confirmed by diagnostic Southern blotting. The haploids were then mated and homozygous mer $1 \Delta$ diploids were selected on YPD agar containing $100 \mu \mathrm{g} / \mathrm{mL}$ nourseothricin and $150 \mu \mathrm{g} / \mathrm{mL}$ geneticin.

\section{Introduction of cDNAs}

Empty pRS305 vector and pRS305-cMER2-cMER3 were linearized by AgeI digestion and transformed, respectively, into haploid wild-type, nam $8 \Delta$, and mer1s SK1 strains (MATa). The Leu ${ }^{+}$transformants were selected and integration of cDNA-containing fragments or empty vector fragments into their leu2::hisG genomic loci was verified by diagnostic PCR. Empty pRS306 vector, pRS306-cAMA1, pRS306-cSPO22, and pRS306-cAMA1-cSPO22 were linearized by StuI digestion and transformed into haploid wild-type, nam $8 \Delta$, and 
mer1 $\Delta$ SK1 strains $(M A T \alpha)$. The $\mathrm{Ura}^{+}$transformants were selected and integration of the CDNA-containing fragments or empty vector fragments into their ura3 genomic loci was verified by PCR. Next, the SK1 MATa and MATa strains were crossed and $\mathrm{Leu}^{+} \mathrm{Ura}^{+}$diploids were selected. Empty pRS305 vector and pRS305-cSAE3 were linearized by AgeI digestion and transformed into wild-type and $\operatorname{tgs} 1 \Delta$ diploid strains. The $\mathrm{Leu}^{+}$transformants were selected and integration of cDNA-containing fragments or empty vector fragments into their leu2::hisG genomic loci was verified by PCR. Wild-type and mutant NAM8-containing pRS305 plasmids were digested with AgeI and inserted into the leu2::his G locus of nam $8 \Delta$ diploid strains.

\section{Sporulation}

Single colonies of diploid yeast strains were patched on YPG agar plates (1\% yeast extract, 2\% bactopeptone, 3\% glycerol, 2\% bactoagar) for at least $6 \mathrm{~h}$ to select for cells with healthy mitochondria. Cells were streaked on YPD agar plates and incubated for $3 \mathrm{~d}$ at $30^{\circ} \mathrm{C}$. Single colonies were then inoculated into YPD liquid medium and grown at $30^{\circ} \mathrm{C}$ to stationary phase $\left(A_{600}\right.$ of $\left.6-8\right)$. Aliquots were inoculated into $12.5 \mathrm{~mL}$ of presporulation medium $(0.5 \%$ yeast extract, $1 \%$ peptone, $0.67 \%$ yeast nitrogen base [without amino acids], $1 \%$ potassium acetate, $0.05 \mathrm{M}$ potassium biphthalate $[\mathrm{pH}$ 5.5], $0.002 \%$ antifoam 204) to attain an $A_{600}$ of 0.8 . The cultures were incubated for $7 \mathrm{~h}$ at $30^{\circ} \mathrm{C}$ and added to $100 \mathrm{~mL}$ of fresh presporulation medium to attain an $A_{600}$ of 0.025 (wild-type, mer1 $\Delta$, and nam $8 \Delta$ cells) or 0.1 (tgs $1 \Delta$ cells). These cultures were incubated for $16 \mathrm{~h}$ until $A_{600}$ reached $\sim 3.0$. The cells were harvested by centrifugation, washed twice with sporulation medium ( $2 \%$ potassium acetate, $0.001 \%$ polypropylene glycol), and then resuspended in sporulation medium at $A_{600}$ of 6 . Aliquots were withdrawn from synchronous meiotic cultures at $6,8,10,12,14$, and $24 \mathrm{~h}$ post transfer to sporulation medium. The cells were fixed in an equal volume of $100 \%$ ethanol and then examined by light microscopy (100x magnification) to assess the abundance of four-spore asci. A total of 200 cells from each sample were scored. The extents of sporulation (\% asci) are plotted as a function of time in Figure 1. Each datum is the average of three separate experiments \pm SEM.

\section{SUPPLEMENTAL MATERIAL}

Supplemental material is available for this article.

\section{ACKNOWLEDGMENTS}

This work was supported by US National Institutes of Health grants GM52470 (S.S.) and GM50288 (B.S.). S.S. is an American Cancer Society Research Professor.

Received April 26, 2011; accepted June 23, 2011.

\section{REFERENCES}

Balzer RJ, Henry MF. 2008. Snu56p is required for Merlp-activates meiotic splicing. Mol Cell Biol 28: 2497-2508.

Cooper KF, Mallory MJ, Egeland DB, Jarnik M, Strich R. 2000. Amalp is a meiosis-specific regulator of the anaphase promoting complex/cyclosome in yeast. Proc Natl Acad Sci 97: 14548-14553.

Engebrecht J, Roeder GS. 1990. MER1, a yeast gene required for chromosome pairing and genetic recombination is induced in meiosis. Mol Cell Biol 10: 2379-2389.
Engebrecht J, Voelkel-Meiman K, Roeder GS. 1991. Meiosis-specific RNA splicing in yeast. Cell 66: 1257-1268.

Fabrizio P, Dannenberg J, Dube P, Kastner B, Stark H, Urlaub H, Lührmann R. 2009. The evolutionarily conserved core design of the catalytic activation step of the yeast spliceosome. Mol Cell 36: 593-608.

Goldstein AL, McCusker JH. 1999. Three new dominant drug resistance cassettes for gene disruption in Saccharomyces cerevisiae. Yeast 15: 1541-1553.

Gottschalk A, Tang J, Puig O, Salgado J, Neubauer G, Colot HV, Mann M, Séraphin B, Rosbash M, Lührmann R, et al. 1998. A comprehensive biochemical and genetic analysis of the yeast U1 snRNP reveals five novel proteins. RNA 4: 374-393.

Juneau K, Palm C, Miranda M, Davis RW. 2007. High-density yeasttiling array reveals previously undiscovered introns and extensive regulation of meiotic splicing. Proc Natl Acad Sci 104: 1522-1527.

Maleki S, Neale MJ, Arora C, Henderson KA, Keeney S. 2007. Interaction between Mei4, Rec114, and other proteins required for meiotic double-strand break formation in Saccharomyces cerevisiae. Chromosoma 116: 471-486.

Munding EM, Igel AH, Shiue L, Dorighi KM, Trevino LR, Ares M. 2010. Integration of a splicing network within the meiotic gene expression program of Saccharomyces cerevisiae. Genes Dev 24: 2693-2704.

Nakagawa T, Ogawa H. 1997. Involvement of the MRE2 gene of yeast in formation of meiosis-specific double-strand breaks and crossover recombination through RNA splicing. Genes Cells 2: 65-79.

Nakagawa T, Ogawa H. 1999. The Saccharomyces cerevisiae MER3 gene, encoding a novel helicase-like protein, is required for crossover control in meiosis. EMBO J 18: 5714-5723.

Nandabalan K, Price L, Roeder GS. 1993. Mutations in U1 snRNA bypass the requirement for a cell type-specific RNA splicing factor. Cell 73: 407-415.

Nilsen TW, Graveley BR. 2010. Expansion of the eukaryotic proteome by alternative splicing. Nature 463: 457-463.

Oelschlaegel T, Schwickart M, Matos J, Bogdanova A, Mamasses A, Havlis J, Shevchenko A, Zachariae W. 2005. The yeast APC/C subunit Mnd2 prevents premature sister chromatid separation triggered by the meiosis-specific APC.C-Ama1. Cell 120: 773-788.

Penkner AM, Prinz S, Ferscha S, Klein F. 2005. Mnd2, an essential antagonist of the anaphase-promoting complex during meiotic prophase. Cell 120: 789-801.

Primig M, Williams RM, Winzeler EA, Tevzadze GG, Conway AR, Hwang SY, Davis RW, Esposito RE. 2000. The core meiotic transcriptome in budding yeast. Nat Genet 26: 415-423.

Qiu ZR, Schwer B, Shuman S. 2011a. Determinants of Nam8dependent splicing of meiotic pre-mRNAs. Nucleic Acids Res 39: 3427-3445.

Qiu ZR, Shuman S, Schwer B. 2011b. An essential role for trimethylguanosine RNA caps in Saccharomyces cerevisiae meiosis and their requirement for splicing of SAE3 and $\mathrm{PCH} 2$ meiotic pre-mRNAs. Nucleic Acids Res doi: 10.1093/nar/gkr083.

Rabitsch KP, Toth A, Galova M, Schleiffer A, Schaffner G, Aigner E, Rupp C, Penkner AM, Moreno-Borchart AC, Primig M, et al. 2001. A screen for genes required for meiosis and spore formation based on whole-genome expression. Curr Biol 11: 1001-1009.

San-Segundo PA, Roeder GS. 1999. Pch2 links chromatin silencing to meiotic checkpoint control. Cell 97: 313-324.

Sickmier EA, Frato KE, Shen H, Paranawithana SR, Green MR, Kielkopf CL. 2006. Structural basis for polypyrimidine tract recognition by the essential pre-mRNA splicing factor U2AF65. Mol Cell 23: 49-59.

Spingola M, Ares M. 2000. A yeast intronic splicing enhancer and Nam8p are required for Merlp-activated splicing. Mol Cell 6: 329-338.

Spingola M, Armisen J, Ares M. 2004. Merlp is a modular splicing factor whose function depends on the conserved U2 snRNP protein Snu17p. Nucleic Acids Res 32: 1242-1250.

Wach A, Brachat A, Pohlmann R, Philippsen P. 1994. New heterologous modules for classical or PCR-based gene disruptions in Saccharomyces cerevisiae. Yeast 10: 1793-1808. 

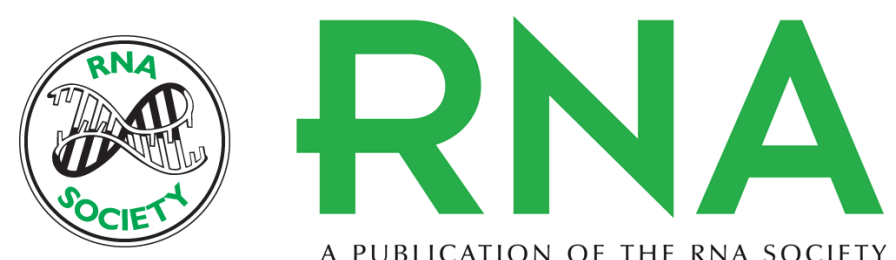

A PUBLICATION OF THE RNA SOCIETY

\section{Defining the Mer1 and Nam8 meiotic splicing regulons by cDNA rescue}

Zhicheng R. Qiu, Beate Schwer and Stewart Shuman

RNA 2011 17: 1648-1654 originally published online July 25, 2011

Access the most recent version at doi:10.1261/rna.2792011

\section{Supplemental http://rnajournal.cshlp.org/content/suppl/2011/07/19/rna.2792011.DC1 \\ Material}

References This article cites 24 articles, 7 of which can be accessed free at:

http://rnajournal.cshlp.org/content/17/9/1648.full.html\#ref-list-1

\section{License}

Email Alerting Receive free email alerts when new articles cite this article - sign up in the box at the Service top right corner of the article or click here.

To subscribe to RNA go to:

http://rnajournal.cshlp.org/subscriptions 